\title{
Caracterização de cuidadores de vítimas de trauma crânio-encefálico em seguimento ambulatorial
}

\author{
CHARACTERIZATION OF CAREGIVERS OF VICTIMS OF TRAUMATIC BRAIN \\ INJURY ON AN OUTPATIENT FOLLOW-UP PROGRAM
}

\author{
CARACTERIZACIÓN DE CUIDADORES DE VÍCTIMAS DE TRAUMA CRÁNEO-ENCEFÁLICO \\ CON SEGUIMIENTO AMBULATORIO
}

\section{Edilene Curvelo Hora', Regina Márcia Cardoso de Sousa ${ }^{2}$, Rocio Elizabeth Chávez Alvarez ${ }^{3}$}

\section{RESUMO}

O presente estudo caracterizou o cuidador familiar principal de 50 vítimas de trauma crânio-encefálico em seguimento ambulatorial, em um centro para atendimento de trauma na cidade de São Paulo, por meio de entrevista e análise de prontuário. Os resultados revelaram que os cuidadores tinham idade média de 44,90 anos, eram em sua maioria do sexo feminino, solteiros, católicos, não haviam completado o ensino fundamental e não tinham ocupação remunerada antes do trauma. A metade dos cuidadores principais era mãe da vítima, $22 \%$ esposa(o) e $18 \%$ irmã(ão). A presença do cuidador secundário foi observada em $48 \%$ dos casos avaliados.

\section{DESCRITORES}

Traumatismos cerebrais.

Cuidadores.

Enfermagem familiar.

\begin{abstract}
The present study characterized the main family caregiver of 50 victims of traumatic brain injury on an outpatient follow-up program of a trauma center in São Paulo through interviews and record analyses. The results revealed that caregivers had average age of 44.9, were mostly female, single, Catholic, had not completed elementary school and had no paid occupation prior to the trauma. One half of the main caregivers was the victim's mother, 22 percent was the spouse and 18 percent was a sibling. The presence of a secondary caregiver was observed in 48 percent of the cases.
\end{abstract}

\section{KEY WORDS}

Brain injuries.

Caregivers.

Family nursing.

\section{RESUMEN}

En el presente estudio se caracterizó al cuidador familiar principal de 50 víctimas de trauma cráneoencefálico con seguimiento ambulatorio, de un centro para atención de traumas en la ciudad de São Paulo, por medio de entrevista y análisis de la historia clínica. Los resultados revelaron que los cuidadores tenían como edad promedio 44,90 años, en su mayoría eran del sexo femenino, solteros, católicos, no habían completado sus estudios básicos y no tenían ocupación remunerada antes del trauma. La mitad de los cuidadores principales era madre de la víctima, $22 \%$ esposa(o) y el $18 \%$ hermano(a). La presencia del cuidador secundario fue observada en $48 \%$ de los casos evaluados.

\section{DESCRIPTORES}

Traumatismos cerebrales.

Cuidadores.

Enfermería de la familia.
* Extraído da Dissertação "Conseqüências do Trauma Crânio-Encefálico para o cuidador familiar", Escola de Enfermagem da Universidade de São Paulo (EEUSP), 2002

1 Enfermeira. Professora assistente do Departamento de Enfermagem da UFS. Doutoranda em enfermagem pela EEUSP. lenehora@usp.br

2 Enfermeira. Professora Associada do Departamento de Enfermagem Médico-Cirúrgica da EEUSP vian@usp.br

3 Enfermeira. Mestre em Enfermagem pela EEUSP jermiangis@hotmail.com 


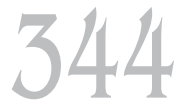

Rev Esc Enferm USP 2005; 39(3):343-9.

\section{INTRODUÇÃO}

No Brasil, os acidentes e a violência configuram um problema de saúde pública de grande magnitude e transcendência, com forte impacto na morbidade e na mortalidade da população ${ }^{(1)}$. No conjunto das lesões decorrentes das causas externas, o Trauma Crânio-Encefálico (TCE) destaca-se em termos de magnitude tanto entre mortos quanto feridos, sendo uma das lesões mais freqüentes ${ }^{(2)}$.

As vítimas que sobrevivem ao TCE podem apresentar deficiências e incapacidades que podem ser temporárias ou permanentes, interferindo na capacidade do indivíduo de desempenhar suas funções $^{(3)}$. Nestes casos, a presença do cuidador no auxílio das vítimas é uma necessidade freqüente.

O cuidador familiar ou informal é alguém da família ou afim, sem formação na área de saúde que está cuidando de um ente familiar e, portanto, pressupõe-se que tenha cumplicidade e compromisso com a pessoa cuidada. Pode ser ele categorizado em primário ou principal e secundário a depender da maior ou menor intensidade em que presta cuidados ao ente familiar ${ }^{(4)}$.

Cuidar não é uma tarefa fácil, exige uma mudança radical na vida de quem cuida, também demanda a execução de tarefas complexas, delicadas e sofridas. Em muitos casos, o cuidador é uma pessoa frágil, já em idade de envelhecimento ou em vias de ficar doente e se não tiver um suporte poderá ser um futuro paciente ${ }^{(5)}$.

Os membros da família com freqüência enfrentam as mudanças ocorridas no paciente com TCE sem suporte ou com suporte insuficiente, especialmente, quando ocorrem a longo prazo mudanças cognitivas e de personalidade ${ }^{(6)}$.

O suporte social, tão necessário a esses cuidadores, é abordado como um processo dinâmico que envolve relacionamentos entre o indivíduo e todo seu sistema social. De acordo com a Academia Nacional de Ciências dos Estados Unidos, existem quatro formas de fornecer esse suporte social: suporte afetivo (qualifica o apoio ao indivíduo sob a forma de empatia, amizade, amor e confiança); suporte de participação (incrementa a capacidade do indivíduo em apoiar outras pessoas em situações em que as mesmas necessitam de ajuda); suporte material ( ajuda direta a uma pessoa, por meio de doação de objetos e contribuição financeira) e suporte de informação (oferece indicações que possam ajudar o indivíduo a resolver seus problemas) $)^{(7)}$.

Conhecer as características da vítima e cuidador, além de suas necessidades individuais é uma etapa inicial para planejar formas de prover o suporte necessário ao binômio cuidador e vítima. Frente a essas considerações é propósito deste estudo caracterizar o cuidador familiar principal de um grupo de vítimas de TCE em seguimento ambulatorial em um hospital de referência para trauma na cidade de São Paulo.

\section{CASUÍSTICA E MÉTODO}

Esta pesquisa caracteriza-se como exploratória, de campo e com abordagem quantitativa. Foi desenvolvida, após aprovação da Comissão de Ética e Pesquisa, no Ambulatório do Trauma de Crânio do Instituto Central do Hospital das Clínicas da Faculdade de Medicina da Universidade de São Paulo (IC-HCFMUSP), centro de referência para atendimento ao trauma na região oeste da Grande São Paulo. A coleta de dados foi realizada no período de junho a dezembro de 2001.

Participaram do estudo 50 cuidadores de vítimas de TCE que atenderam aos seguintes critérios: comparecer ao Ambulatório, em decorrência ao acompanhamento da vítima; ter idade igual ou superior a 18 anos e cuidar de vítimas de TCE na faixa etária entre 12 e 60 anos; ser cuidador familiar principal da vítima de TCE que sofreu evento traumático há pelo menos seis meses.

As vítimas de TCE, que tinham cuidadores que atendiam aos critérios anteriormente descritos foram incluídas. A participação do cuidadore da vítima na casuística da presente investigação foi vinculada ao consentimento verbal e escrito de ambos.

A coleta de dados foi realizada, por meio de entrevista com a vítima e seu cuidador, além da análise de prontuário. Foram utilizados três instrumentos, o termo de consentimento livre e esclarecido e os formulários para entrevistas com o cuidador e a vítima de TCE que continham questões estruturadas para caracterização de ambos.

No formulário da vítima de TCE havia também uma avaliação da incapacidade e dependência, por meio da Escala de Resultados de Glasgow (ERG). Essa escala criada por Jennett e Bond em $1975^{(8)}$, classifica as vítimas de TCE, segundo sua capacidade funcional. A versão original estabelece cinco categorias: morte, estado vegetativo persistente (não obedece aos comandos simples nem pronuncia qualquer palavra), incapacidade grave (consciente, mas dependente), incapacidade moderada (independente, mas incapaz) e boa recuperação (retomam a vida normal, embora possam persistir leves deficiências físicas ou mentais). 
Foi feita uma análise de todos os prontuários das vítimas agendadas para atendimento, realizando-se dessa forma uma triagem inicial por meio da data do trauma e idade da vítima. Os dados de caracterização existentes no prontuário foram registrados em formulário e os cuidadores foram abordados, sendo realizado uma entrevista estruturada e individual.

Os dados foram armazenados em um banco de dados computadorizado, construído com o programa SPSS 8.0 e apresentados em distribuição de freqüências, sob a forma de tabelas. Na análise descritiva das variáveis quantitativas foi apresentada estatística descritiva (média, desvio-padrão, mínimo, mediana e máximo).

$\mathrm{Na}$ elaboração desse banco de dados, as causas externas descritas no prontuário e confirmadas na entrevista foram agrupadas, segundo a Classificação Estatística Internacional de Doenças e problemas relacionados à Saúde da Organização Mundial da Saúde ${ }^{(9)}$.
A classificação da ocupação principal do cuidador, antes do trauma de seu familiar, foi baseada nos níveis ocupacionais recomendados no estudo $^{(10)}$, descrita em três níveis a seguir: ocupações manuais não especializadas (não exigem experiência profissional prévia), ocupações manuais especializadas (exigem conhecimento completo e detalhado dos processos que intervêm no trabalho, com elevado grau de habilidade manual) e outras ocupações (envolvem tarefas não manuais e que exigem níveis mínimos de escolaridade e ou experiência prévia e inclui os cargos de supervisão, coordenação e técnico de nível médio).

\section{RESULTADOS}

Nas Tabelas 1 e 2 são apresentadas as características clínicas e demográficas de cuidadores e vítimas. A análise conjunta desses dois grupos é sempre imprescindível quando a proposta é o suporte familiar após o trauma.

Tabela 1-Características dos cuidadores principais (n=50), IC-HCFMUSP. (São Paulo, 2001)

\begin{tabular}{|c|c|}
\hline \multicolumn{2}{|l|}{ Sexo } \\
\hline Feminino & $88 \%$ \\
\hline Masculino & $12 \%$ \\
\hline \multicolumn{2}{|l|}{ Idade (anos) } \\
\hline Média & 44,90 \\
\hline Desvio Padrão & 13,19 \\
\hline Mediana & 46 \\
\hline Variação & $20-75$ \\
\hline \multicolumn{2}{|l|}{ Situação Conjugal } \\
\hline Solteiro(a) & $68 \%$ \\
\hline Casado(a) & $28 \%$ \\
\hline Amasiado(a) & $4 \%$ \\
\hline \multicolumn{2}{|l|}{ Religião } \\
\hline Católica & $58 \%$ \\
\hline Evangélica & $30 \%$ \\
\hline Espírita & $4 \%$ \\
\hline Outras & $2 \%$ \\
\hline Não tem & $6 \%$ \\
\hline \multicolumn{2}{|l|}{ Escolaridade } \\
\hline Analfabeto & $4 \%$ \\
\hline Fundamental incompleto & $60 \%$ \\
\hline Fundamental completo & $16 \%$ \\
\hline Médio incompleto & $6 \%$ \\
\hline Médio completo & $12 \%$ \\
\hline Superior completo & $2 \%$ \\
\hline \multicolumn{2}{|c|}{ Ocupação remunerada antes do trauma } \\
\hline Manuais não especializadas & $32 \%$ \\
\hline Manuais especializadas & $16 \%$ \\
\hline Outras & $16 \%$ \\
\hline Não tinha & $36 \%$ \\
\hline \multicolumn{2}{|l|}{ Parentesco com as vítimas } \\
\hline Mãe & $50 \%$ \\
\hline Esposo(a) & $22 \%$ \\
\hline $\operatorname{Irmão(ã)}$ & $18 \%$ \\
\hline Tio(a) & $4 \%$ \\
\hline Pai & $4 \%$ \\
\hline Filho(a) & $2 \%$ \\
\hline \multicolumn{2}{|c|}{ Ajuda de cuidadores secundários } \\
\hline Sim & $48 \%$ \\
\hline Não & $52 \%$ \\
\hline
\end{tabular}

Caracterização de cuidadores de vítimas de trauma crânio-encefálico em seguimento ambulatorial 
Edilene Curvelo Hora Regina Márc ia C. de Sousa Rocio Elizabeth C. Alvarez
Na distribuição dos cuidadores e vítimas quanto ao sexo, observa-se que a grande maioria dos cuidadores era do sexo feminino (88\%) e das vítimas, do sexo masculino (90\%). A idade média das vítimas foi 30,60 anos com desvio-padrão igual a 12,08 , valores menores que os relativos a idade dos cuidadores, 44,90 e 13,19, respectivamente.

Tabela 2-Características das vítimas (n=50), IC-HCFMUSP. (São Paulo, 2001)
Quanto ao tempo decorrido após o trauma que os cuidadores e vítimas foram entrevistados, verifica-se que o tempo médio do trauma das vítimas foi 4,42 anos com desvio-padrão igual a 4,82 , atingindo o tempo máximo do trauma igual a 18,4 anos.

$\begin{array}{lc}\text { Sexo } & \\ \text { Feminino } & 90 \% \\ \text { Masculino } & 10 \% \\ \text { Idade (anos) } & 30,60 \\ \text { Média } & 12,08 \\ \text { Desvio Padrão } & 27 \\ \text { Mediana } & 13-60 \\ \text { Variação } & \\ \text { Tempo do trauma(anos) } & 4,42 \\ \text { Média } & 4,82 \\ \text { Desvio-Padrão } & 0,58-18,40 \\ \text { Variação } & \\ \text { Causa externa } & 74 \% \\ \text { Acidentes de transporte } & 16 \% \\ \text { Quedas } & 10 \% \\ \text { Agressões } & \\ \text { Nível de recuperação pós-trauma } & 56 \% \\ \text { Incapacidade grave } & 14 \% \\ \text { Incapacidade moderada } & 30 \% \\ \text { Boa recuperação } & \\ \text { Situação Conjugal } & 46 \% \\ \text { Casado(a) } & 22 \% \\ \text { Viúvo(a) } & 14 \% \\ \text { Amasiado(a) } & 12 \% \\ \text { Separado(a) } & 6 \% \\ \text { Solteiro(a) } & \end{array}$

Observa-se que a maioria das vítimas apresentou acidentes de transporte como causa externa do TCE (74\%) e a minoria foi vítima de quedas $(16 \%)$ ou agressões (10\%). A distribuição desse grupo quanto a recuperação pós-trauma revela que a maioria (56\%) apresentou incapacidade grave, $30 \%$ demonstraram boa recuperação e a minoria, incapacidade moderada. Vítimas extremamente dependentes, em estado vegetativo persistente, não foram identificadas.

Os dados apresentados quanto à religião revelam que $58 \%$ dos cuidadores eram católicos, $30 \%$ evangélicos e poucos informaram não ter religião.

A distribuição dos cuidadores quanto à escolaridade mostra que a maioria dos cuidadores apresentou ensino fundamental incompleto $(60 \%)$ e somente $20 \%$ da casuística analisada chegou a ingressar no ensino médio ou a freqüentar o nível superior.

Antes do trauma, 36\% dos cuidadores não exerciam ocupação remunerada. Entre os demais, metade tinha ocupações manuais não especializadas.
Quanto à situação conjugal, a maioria dos cuidadores era solteira (68\%) e a minoria era casada ou amasiada (32\%). Entre as vítimas, 60\% eram casadas ou amasiadas, com viúvas, solteiras e separadas representando $40 \%$ do grupo estudado.

A distribuição do grau de parentesco dos cuidadores com as vítimas revela que a metade dos incluídos no estudo era mãe da vítima; a outra metade, na maioria era esposo(a) ou irmão(ã).

Todos os cuidadores em estudo eram os principais responsáveis pelas respectivas vítimas, e $48 \%$ deles tinham alguém para ajudar nos cuidados. Na distribuição dos ajudantes (cuidadores secundários) a maioria era filho(a) e mãe, seguida de esposo(a) e irmão(a); outros graus de parentesco foram muito pouco freqüentes .

\section{DISCUSSÃO}

As características dos cuidadores e vítimas deste estudo foram em vários aspectos semelhantes às encontradas na literatura, embora nenhum estudo nacional sobre cuidadores de vítimas de TCE tenha sido localizado. 
Dos 50 cuidadores estudados, a grande maioria era do sexo feminino $88 \%$, assim como em outros estudos internacionais relacionados a cuidadores de vítimas de $\mathrm{TCE}^{(11-14)}$. Nos estudos nacionais relacionados a cuidadores de pessoas com outras patologias, ocorreu o mesmo ${ }^{(15-17)}$. Existe uma tradição familiar para que o cuidador seja mulher, embora a mesma encontre-se, geralmente, sobrecarregada com outras tarefas ${ }^{(5)}$.

Por outro lado, como já foi bem documentado na literatura, as vítimas de TCE são predominantemente do sexo masculino. Nas 50 vítimas de TCE estudadas, os homens eram a grande maioria, $90 \%$. Investigações direcionadas ao binômio vítima e família também vêm documentando a mais alta freqüência desse gênero entre as vítimas ${ }^{(11-14,18)}$.

A idade média dos cuidadores foi 44,90 anos, assemelhando-se à observada em estudos com cuidadores de vítima de TCE em que a média de idade variou entre 43 a 53,29 anos ${ }^{(11-14,19-22)}$. Observações semelhantes foram realizados em estudos nacionais relacionados a outras patologias ${ }^{(15-17)}$.

A idade média das vítimas na época da entrevista era 30,60 anos, com um tempo de trauma médio de 4,42 anos. Assim como em outras publicações, a média de idade das vítimas era inferior a dos cuidadores, mesmo sendo o tempo de trauma médio superior ao do presente estudo, 5,71 a 8,2 anos, observando-se vítimas com tempo de trauma de $34 \operatorname{anos}^{(13-14,20,23)}$. No atual estudo, a mediana da idade dos cuidadores foi 46 anos, com idade máxima de 75 anos, ou seja, pessoas de meia idade ou já idosas eram cuidadores freqüentes.

A grande maioria das vítimas (74\%) apresentou como causa externa o acidente de transporte (atropelamento, acidente automobilístico, acidente de motocicleta, entre outros). Segundo o Consenso da Conferência Americana sobre reabilitação de pessoas vítimas de TCE, cerca de $50 \%$ dos casos são decorrentes de acidentes de veículo a motor, bicicletas e pedestres. As quedas são a segunda causa mais freqüente de TCE entre os idosos e crianças ${ }^{(24)}$.

A maioria das vítimas (56\%) apresentou incapacidade grave, isto é, eram dependentes de outros para realizar atividades básicas para manutenção da vida diária. Os demais eram independentes, porém apresentavam incapacidade que os restringia de realizar algumas atividades (incapacidade moderada) ou apresentavam boa recuperação, ainda que tivessem deficiências que dificultavam a execução de algumas tarefas. A atual investigação aponta para a necessidade de cuidadores de víti- mas de TCE, mesmo quando a independência para manutenção das atividades da vida diária é alcançada. Nesse sentido, outros autores que avaliaram cuidadores de vítimas de TCE, 6 meses e 1 ano após o trauma, notaram predomínio de cuidadores de vítimas com boa recuperação, 51\% e $44 \%$, respectivamente ${ }^{(11-12)}$.

A maioria dos cuidadores era de religião católica $58 \%$, o que se assemelha a resultados sobre cuidadores de vítimas de TCE, $42 \%{ }^{(20)}$ e a outros estudos com cuidadores de patologias diversas $^{(15-17)}$.

A baixa escolaridade observada na maioria dos cuidadores, pode ser relacionada à escolaridade da população brasileira, em geral, ao hospital campo de pesquisa que atende as classes sociais menos favorecidas e a indicação de estudos que a incidência de TCE é maior em famílias de menor renda $^{(3,25)}$.

Quando se fez a somatória de todos os tipos de ocupações apresentadas, a maioria dos cuidadores tinha ocupações remuneradas antes do trauma de seu familiar $64 \%$, entre essas, as mais freqüentes foram as ocupações "manuais não especializadas", $32 \%$, sendo esse resultado compatível com o grau de escolaridade apresentado pelos cuidadores.

Em relação à situação conjugal, a maioria dos cuidadores era solteira $68 \%$, o que difere dos estudos pesquisados, com cuidadores de vítimas de TCE ou de outras doenças. Neles o percentual de casadas permaneceu entre 72 e $85,71 \%{ }^{(13,15-17,20)}$. Em nosso meio, pessoas solteiras, com baixa escolaridade, sem ocupação ou com ocupação não especializada podem ter a preferência da família para assumir o papel de cuidador pelos seguintes motivos: os solteiros, pela menor responsabilidade na estrutura familiar e os que têm menor escolaridade e ocupações pouco definidas, pela dificuldade em se inserir no mercado de trabalho.

Quanto às vítimas, a grande maioria era casada ou amasiada (60\%) na época do trauma. Em 1995, um estudo realizado no mesmo serviço ${ }^{(3)}$ caracteri- $^{-}$ zou as vítimas como homens jovens, predominantemente, solteiros. A condição de solteiro pode minimizar o dano social, pois ocasiona, geralmente, alterações menos radicais na estrutura familiar. Por outro lado, quando a vítima é casada, a sobrecarga da família recai inteiramente só para um dos cônjuges, portanto, há um maior conflito de papéis e dificuldades emocionais, perante as mudanças ocorridas $^{(26)}$.

De forma similar a outros estudos com vítimas de TCE, metade dos cuidadores eram mães das ví-
Caracterização de cuidadores de vítimas de trauma crânio-encefálico em seguimento ambulatorial

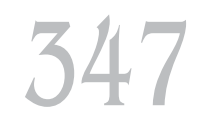

Rev Esc Enferm USP 2005; 39(3):343-9. 
Edilene Curvelo Hora Regina Márc ia C. de Sousa Rocio Elizabeth C. Alvarez timas. Na segunda posição, com $22 \%$ estavam os(as) esposos(as). Os cônjuges alternam com as mães o papel de cuidador principal, portanto, diversamente ao presente estudo, alguns autores apontaram o predomínio dos(as) esposos(as) entre os cuidadores ${ }^{(15,19)}$.

Dos 50 cuidadores do estudo, 24 (48\%) tinham ajuda em sua maioria dos filhos ou mães. Tais resultados reforçam a importância das mães como cuidadoras, pois quando não estavam desempenhando o cuidado principal, estavam no cuidado secundário.

É interessante observar que embora $60 \%$ das vítimas sejam casadas ou amasiadas, na época do trauma, somente $22 \%$ dos cônjuges eram cuidadores principais e $13 \%$ eram cuidadores secundários, o que sugere mudanças no relacionamento conjugal.

O relacionamento marital e sexual de 11 casais heterossexuais foi investigado no período de um a sete anos após o parceiro do sexo masculino ter sofrido TCE grave. Nesse estudo, as mulheres informaram ter reduzida satisfação marital e sexual após o evento traumático(27).

A análise sobre as mudanças nos relacionamentos após TCE constatou que a separação ou divórcio ocorreu em $49 \%$ das vítimas analisadas, no período de cinco a oito anos, após o trau$\mathrm{ma}^{(28)}$. As esposas passam por uma mudança de papel, de esposa e companheira para cuidadora e, muitas vezes, sentem-se morando com uma pessoa "estranha", em virtude das mudanças comportamentais da vítima ${ }^{(29)}$. Investigações têm relatado que os cônjuges sofrem mais que os pais como cuidadores ${ }^{(30)}$, talvez porque os pais freqüentemente têm o suporte do parceiro com quem podem dividir a sobrecarga do cuidado, ou por já terem incorporado o papel de cuidador dos filhos em diferentes fases da vida.

\section{CONSIDERAÇÕES FINAIS}

O estudo com cuidadores familiares vem despertando interesse em muitos pesquisadores, sendo considerado uma área crescente na saúde. Apesar desse desenvolvimento, ainda são reduzidas as publicações referentes ao assunto em nosso meio. Estudos relacionados ao cuidador familiar da vítima de TCE, não foram encontradas na literatura nacional, em contrapartida, na pesquisa internacional, muito se tem estudado o que proporcionou a comparação dos resultados, embora também houvesse uma boa contribuição das investigações desenvolvidas em âmbito nacional, relacionados ao cuidador de vítimas com outros traumas ou enfermidades.

O TCE tem sido considerado a causa mais importante de incapacidade entre jovens e a mais freqüente causa neurológica de morbidade. Em conseqüência, urge a necessidade de profissionais da saúde com conhecimento específico sobre a assistência não só das vítimas, como também dos cuidadores, afim de melhor lidar com esses familiares, ajudando-os a enfrentar as conseqüências adversas, oriundas desse tipo de trauma.

Espera-se que os resultados do presente estudo forneçam subsídios à assistência aos cuidadores familiares que necessitam ser vistos pelos profissionais como clientes que possuem características e necessidades especiais.

\section{REFERÊNCIAS}

(1) Ministério da Saúde. Secretaria de Políticas de Saúde. Política nacional de redução da morbimortalidade por acidentes e violência. Rev Saúde Pública 2000; 34(4):427-30.

(2) Koizumi MS, Lebrão ML, Mello Jorge MHP, Primerano V. Morbimortalidade por traumatismo crânio-encefálico no município de São Paulo. Arq Neuro Psiquiatr 2000; 58(1):81-9.

(3) Sousa RMC. Padrão de recuperação das vítimas de trauma crânio-encefálico aos 6 meses e 1 ano. [tese]. São Paulo (SP): Escola de Enfermagem USP 1995
(4) Faro ACM. Cuidar do lesado medular em casa: a vivência singular do cuidador familiar. [livredocência]. São Paulo (SP): Escola de Enfermagem da USP; 1999.

(5) Leal MGS. O desafio da longevidade e o suporte ao cuidador. Terceira Idade 2000; 11(20):19-29.

(6) Tyerman A, Booth J. Family interventions after traumatic brain injury: a service example. Neurorehabilitation 2001; 16(1):59-66.

(7) Morgado AF, Lovis GM, Milanil I, Caetano G, Abelha L. Suporte social e distúrbios psiquiátricos: em que base se alicerça a associação? Inf Psiquiatr 1996; 15(2):65-8. 
(8) Jennett B, Bond M. Assessment of outcome after severe brain damage. Lancet 1975; 1(7905): 480-4.

(9) Organização Mundial da Saúde (OMS). Manual de classificação estatística internacional de doenças e problemas relacionados à saúde. $8^{\mathrm{a}}$ ed. São Paulo: Centro Colaborador da OMS para Classificação de Doenças em Português/ USP; 2000.

(10) Fonseca GT. Modelo para uma classificação de ocupações. Rev Bras Est Pedag 1967; 47(106): 274-312.

(11) Marsh NV, Kersel DA, Havill JH, Sleigh JW. Caregiver burden at 6 months following severe traumatic brain injury. Brain Inj 1998; 12(3): 225-38.

(12) Marsh NV, Kersel DA, Havill JH, Sleigh JW. Caregiver burden at 1 year following severe traumatic brain injury. Brain Inj 1998; 12(12): 1045-59.

(13) Kosciulek JF, Lustig DC. Differentiation of three brain injury family types. Brain Inj 1999; 13 (4):245-54.

(14) Kolakowsky-Hayner SA, Miner KD, Kreutzer JS. Long-term life quality and family needs after traumatic brain injury. J Head Trauma Rehabil 2001; 16(4):374-85.

(15) Guerra CICO. Fatores de risco para o desgaste do cuidador familiar de paciente na fila de transplante cardíaco. [dissertação]. São Paulo (SP): Escola de Enfermagem USP; 2000.

(16) Perlini NMOG. Cuidar de pessoa incapacitada por acidente vascular cerebral no domicilio: o fazer do cuidador familiar. [dissertação]. São Paulo (SP): Escola de Enfermagem USP; 2000.

(17) Faro ACM. Atividades realizadas no domicílio pelo cuidador familiar da pessoa com lesão medular. Rev Paul Enferm 2001; 20(2):33-43.

(18) Wallace CA, Bogner J, Corrigan JD, Clinchot D, Mysiw WJ, Fugate LP. Primary caregivers of persons with brain injury: life change 1 year after injury. Brain Inj 1998; 12(6):483-93.

(19) Hall KM, Karzmark P, Stevens M, Englander J, O'hare P, Wright J. Family stressors in traumatic brain injury: a two- year follow-up. Arch Phys Med Rehabil 1994; 75(8):876-84.
(20) Kosciulek JF. Relationship of family schema to family adaptation to brain injury. Brain Inj 1997; 11(11):821-30.

(21) Frosch S, Gruber A, Jones C, Myers S, Noel E, Westerlund A. The long term effect of traumatic brain injury on the roles of caregivers. Brain Inj 1997; 11(12):891-906.

(22) Perlesz A, Kinsella G, Crowe S. Psychological distress and family satisfaction following traumatic brain injury: injured individuals and their primary, secondary, and tertiary carers. J Head Trauma Rehabil 2000; 15( 3):909-29.

(23) Knight RTG, Devereux R, Godfrey H. Caring for a family member with a traumatic brain injury. Brain Inj 1998; 12(6):467-81.

(24) National Institutes Health (NIH). Consensus Development Panel on Rehabilitation of Persons with Traumatic. Brain Injury. JAMA 1999; 282(10):974-83.

(25) Kraus JF. Epidemiology of head injury. In: Cooper PR. Head injury. $3^{\text {rd }}$ ed. New York: Williams \& Wilkins; 1993. cap. 1, p. 1-25.

(26) Oddy M, Coughlan T, Tyerman A, Jenkis D. Social adjustment after closed head injury: a further follow-up seven years after injury. J Neurol Neurosurg Psychiatry 1985; 48(6):564-8.

(27) Gosling J, Oddy M. Rearranged marriages: marital relationships after head injury. Brain Inj 1999; 13(10):785-96.

(28) Wood RL, Yurdakul LK. Change in relationship status following traumatic brain injury. Brain Inj 1999; 13(10):785-96.

(29) Acorn S, Roberts E. Head injury: impact on the wives. J Neurosc Nurs 1992; 24(6):324-8.

(30) Kreutzer JS, Marwitz JH, Kepler K. Traumatic brain injury: family response and outcome . Arch Phys Med Rehabil 1992; 73(8):771-8.
Caracterização de cuidadores de vítimas de trauma crânio-encefálico em seguimento ambulatorial
Correspondência: Edilene Curvelo Hora Rua Alves Guimarães, 461 Ap. 134 - Jd. América CEP -05410-000 - SP 